\title{
Magniezia gardei n.sp. (Crustacea Isopoda Asellota): un Sténasellide des eaux souterraines du Maroc sud-oriental
}

\author{
par
}

Guy MAGNIEZ*

\section{SUMMARY}

Magniezia gardei n.sp. (Crustacea Isopoda Asellota): a Stenasellid from the underground waters of Southeastern Morocco.

This new species has been discovered in the waters of Kef Aziza Cave, on the Saharian slopes of the High Atias Range, Southeastern Morocco. Magniezia gardei n.sp. belongs to the same Genus as four species previously described from the phreatic waters of the Guinean area. So, it is assumed that this Moroccan species should be a relict of the ancient aquatic hypogean fauna of the entire West Africa, nowadays protected by the development of an arid climate.

Origine du matériel: le 22 août 1977, plusieurs spéléologues de Barcelone ont exploré la grotte Kef Aziza, située à proximité de la localité de Tazouguerte (ou Tazzouguert), à environ $25 \mathrm{~km}$ au N-W de Boudenib, en remontant la vallée de l'Oued Guir, sur le versant saharien du Haut-Atlas marocain. Dans les collections d'eau de la cavité (température: $14^{\circ} \mathrm{C}$ ), de grands Asellotes furent observés et capturés. Tous les individus collectés sont spécifiquement identiques et appartiennent à une nouvelle espèce de Sténasellide que nous avons dédiée à $M$. Arturo Garde, en raison de l'intérêt qu'il prend à la recherche de la faune cavernicole. Cette espèce se range sans hésitation dans le genre Magniezia, défini par Lanza en 1966, pour des Sténasellides des eaux souterraines de Côte d'Ivoire, de Guinée-Bissau, du Sénégal et du Mali. Nous remercions très sincèrement MM. Juan Abad, Arturo Garde et Angel Lagar pour la confiance qu'ils nous témoignent en nous confiant ce matériel.

Lot capturé: 9 adultes dont 6 de de 11,7-11,5-11-10,5-10,4 et 10,1 mm environ et $3 \%$ de 14,5-13,9 et $11,8 \mathrm{~mm}$ environ. La 9 de $13,9 \mathrm{~mm}$ possède un marsupium vide (fin d'intermue de reproduction), tandis que les 2 autres sont en intermue

*) Laboratoire de Biologie Animale et Générale, Université de Dijon, 6, Bd. Gabriel, 21100 DIJON, FRANCE. 
de repos génital (présence d'oostégites non fonctionnels subquadrangulaires, de petite taille, plus longs que larges et n'atteignant pas la ligne médiane ventrale du corps). Le $\widehat{o}$ de 11,7 et la + de $14,5 \mathrm{~mm}$ ont été choisis respectivement comme holotype et allotype de Magniezia gardei n.sp. et la description s'appuie sur l'observation de l'ensemble du lot.

Caractères généraux: aspect assez semblable à celui de Magniezia africana ou de $M$. laticarpa. Corps robuste et tégument bien induré. Coefficient d'allongement supérieur à 5 et corps à bords sensiblement parallèles. Largeurs respectives de la tête, des 7 péréionites libres, des 2 pléonites libres et du pléotelson chez le $\hat{o}$ de 10,4 mm: 1,54-1,83-1,99-2,01-1,99-1,88-1,94-1,93-1,78-1,73 et $1,64 \mathrm{~mm}$. Céphalon globuleux à marge rostrale nettement concave (fig. 12). Péréionite I avec les angles antérieurs séparés du bouclier tergal par un sillon articulaire chez les $Q$ s'étant déjà reproduites (cf. Magniez, 1975, p. 123). Péréionites I et V nettement plus courts que les autres. Pléonites I et II très développés en longueur (fig. 17) comme chez les autres Magniezia, avec des pointes pleurales très marquées. Pléotelson subquadrangulaire nettement plus long que large $(2,41 \times 1,64 \mathrm{~mm})$ chez l'individu précité. Pointe caudale atténuée (fig. 17).

Appendices céphaliques: antennules aussi longues que la hampe de l'antenne $(q)$, un peu plus longues ( $\delta$ ). Hampe de 4 articles. Fouet de 18 articles au maximum chez le $\hat{\delta}$, les articles 6 à 17 portant chacun une lame olfactive de $135 \mu$ environ. 14 articles et 11 lames olfactives chez la $q$ (fig. 6). Antennes avec hampe de 6 articles dont le 3ème porte sur sa marge externe une squama réduite, mais munie de 2 fortes soies (fig. 7). Fouet avec 50-60 articles chez le $\hat{\delta}$, moins chez la 9 . Mandibules typiques de Sténasellide, avec corps coudé pratiquement à $90^{\circ}$ et palpe triarticulé normal. Processus inciseur à 4 dents très indurées. Lacinia mobilis de la mandibule gauche fortement développée et possèdant également 4 dents, suivie d'une rangée de 18 tiges (20 à droite) dentelées unilatéralement sur la moitié distale de leur marge rostrale. Lobe molaire peu important et garni de 10-15 soies simples de longueurs variées. Maxillules: lobe externe portant, sur deux rangées distales, 9 lames denticulées et 4 tiges rameuses de longueur croissant de 65 à $145 \mu$ vers l'extérieur. Lobe interne avec 5 épines rameuses ou semidenticulées, dont 2 de $95-105 \mu$ et 3 de $150 \mu$.

Maxilles trilobées normales. Lobes externe et moyen portant chacun une rangée distale d'une dizaine de lames falciformes dentelées sur leur marge interne, de longueur décroissante de l'extérieur vers l'intérieur (respectivement de 260 à $95 \mu$ et de 205 à $65 \mu$ ). Lobe interne entier avec 12-13 tiges rameuses polymorphes distales $(115-80 \mu)$, implantées sur deux rangs. Marge interne avec une quinzaine de très fines sétules lisses.

Maxillipèdes: typiques de Sténasellide. Pas d'épipodite, rétinacle à 3-4 crochets, palpe à 5 articles garnis de soies lisses sur leurs marges internes. Chez la $q$ à marsupium, la base porte un oostégite membraneux réniforme de $930 \mu$ de long et $400 \mu$ de large, glabre, sauf sur ses marges convexes qui portent des herses de fines sétules de 5-6 $\mu$ de long (fig. 4). 
Péréiopodes I: ils constituent de puissants gnathopodes avec une musculature imposante. Marge sternale du carpopodite portant un bouquet de fortes tiges ensiformes dirigées face à l'ongle. Marge sternale du propodite armée de deux rangées de fortes épines dont le bord distal est muni d'une brosse de fines ramifications. Entre ces deux rangées vient se rabattre le dactylopodite dont la marge sternale est constituée par une rangée continue de quelques 15 lames tranchantes qui sont des épines sternales aplaties (fig. 1, 2, 3).

Péréiopodes II à VII: robustes, avec une chétotaxie surtout dense au niveau de la marge sternale des carpopodites II, III et IV. Dactylopodites à 2 fortes épines sternales (irrégulièrement 3 sur certains péréiopodes postérieurs des plus grands individus). Longueurs relatives des péréiopodes I à VII chez le $\widehat{\delta}$ de 10,4 mm: 0,99-1-1,04-0,96-1,13-1,28 et 1,39. Basipodite des péréiopodes II à VII avec une carène tergale très marquée, portant une rangée marginale de 7-8 soies sensorielles "en palmier" dépassant parfois $300 \mu$ de long et alternant avec de courtes tiges lisses banales. Une paire de papilles génitales de 700-800 $\mu$ de long et $200 \mu$ de diamètre sont issues de l'angle interne des coxopodites des péréiopodes postérieurs du $\widehat{\partial}$.

Pléopodes II $q$ : ils présentent une suture secondaire sur le 1/6ème proximal de leur marge interne (fig. 8). La face sternale porte 7-8 soies lisses; la marge distale 3 tiges légèrement plumeuses et la marge externe une soie exopodiale simple.

Pléopodes I ô : protopodite subquadrangulaire sans rétinacle. Exopodite presque 2 fois plus long que large, avec une rangée marginale continue de 20-25 soies lisses, décroissant de la marge interne à la marge externe (fig. 9).

Pléopodes II $\delta$ : ils sont très caractéristiques du genre Magniezia. Le protopodite, subquadrangulaire est très allongé et dirigé obliquement vers l'extérieur. Il contient une très puissante musculature motrice des deux rames. Exopodite biarticulé, avec article proximal très court, mais article distal extrêmement allongé et garni d'une herse distale de 7 soies simples. Endopodite biarticulé avec article proximal long, mais robuste. Les articulations protopodite-premier article et premier-second articles sont fonctionnelles et permettent à la rame d'effectuer des mouvements de grande amplitude dans le plan général de l'appendice (fig. 10).

Article distal falciforme, presque plan, mais épais, formé d'une lame principale terminée par un éperon épais et induré (e) sur laquelle se replie une lamelle accessoire (1). L'ensemble détermine la formation d'un court canal, avec orifice supérieur afférent et orifice inférieur efférent très larges. Cette architecture très primitive de l'organe copulateur est caractéristique du genre Magniezia, mais ici, l'organe semble un peu plus évolué que celui de l'espèce-type $M$. africana (fig. 11).

Pléopodes III: exopodite subquadrangulaire induré, très allongé (fig. 13). Endopodite entier, régulièrement ovalaire, charnu et respiratoire. 


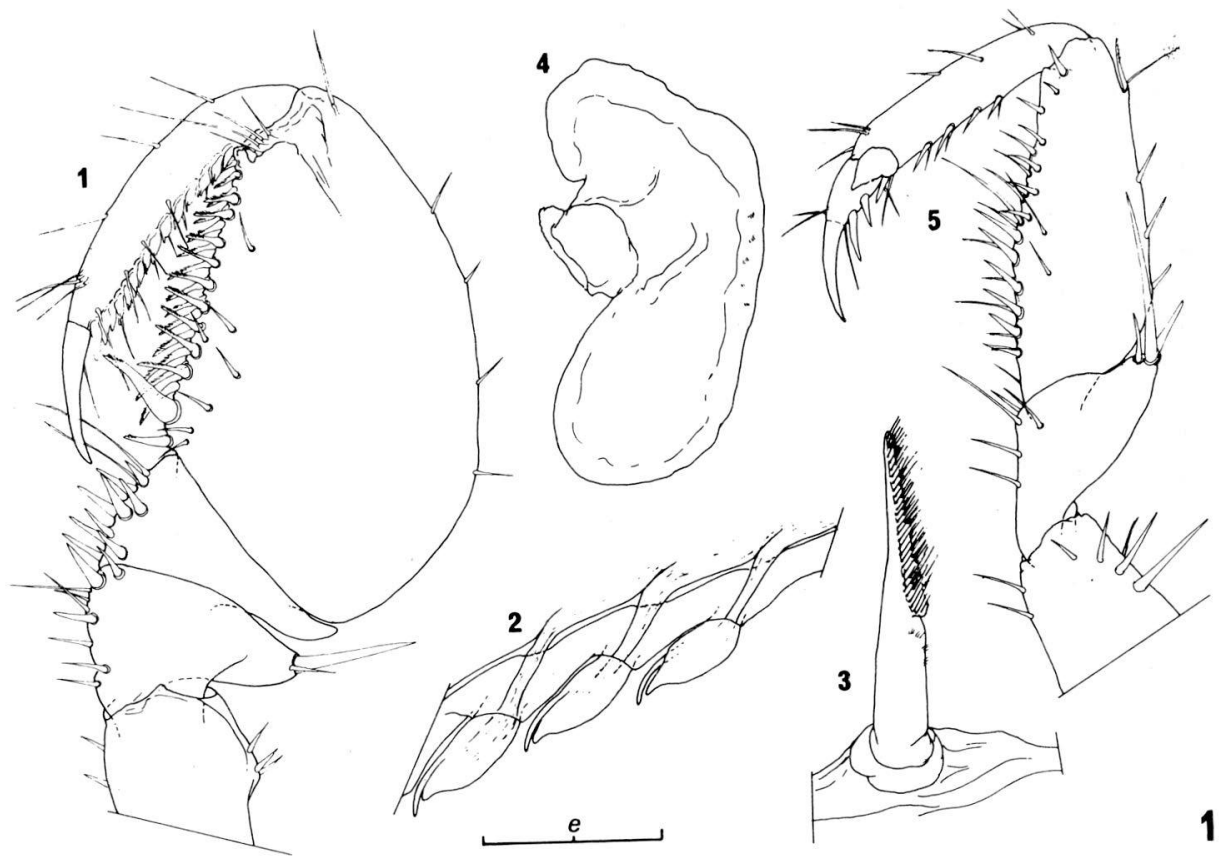

Fig. 1. Péréiopode I droit, ôde $10,4 \mathrm{~mm}$; e $=400 \mu$

Fig. 2. Détail de la marge sternale du dactylopodite du précédent: armature de lames digitées; $\mathrm{e}=66 \mu$

Fig. 3. Tige de l'armature sternale du propodite de 1 ; e $=66 \mu$

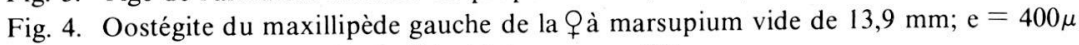

Fig. 5. Péréiopode II gauche du ôde $10,4 \mathrm{~mm}$; $=400 \mu$

Pléopodes IV: exopodite subovalaire induré, très grand, avec une suture interarticulaire très oblique. Il porte une rangée continue d'environ 25 fines soies plumeuses réparties sur la marge interne. Endopodite ovalaire entier, charnu et respiratoire (fig. 14).

Pléopodes V: exopodite ovalaire, lamelleux et induré avec suture interarticulaire moins oblique que celle du IV (fig. 15). Une aire portant des écailles cuticulaires auriculées (fig. 16) dans la zone distale-interne. Endopodite ovalaire, entier, charnu et respiratoire.

Uropodes: pratiquement aussi longs que le pléotelson ( 2,41 et $2,30 \mathrm{~mm}$ chez le $\widehat{o}$ de $10,4 \mathrm{~mm}$ ). Longueur de l'exopodite égale aux 8/9èmes de celle de l'endopodite. Les deux rames portent un pinceau de soies simples dont les plus longues dépassent $800 \mu$ (fig. 18).

Le genre Magniezia Lanza: il comprend désormais 5 espèces: 


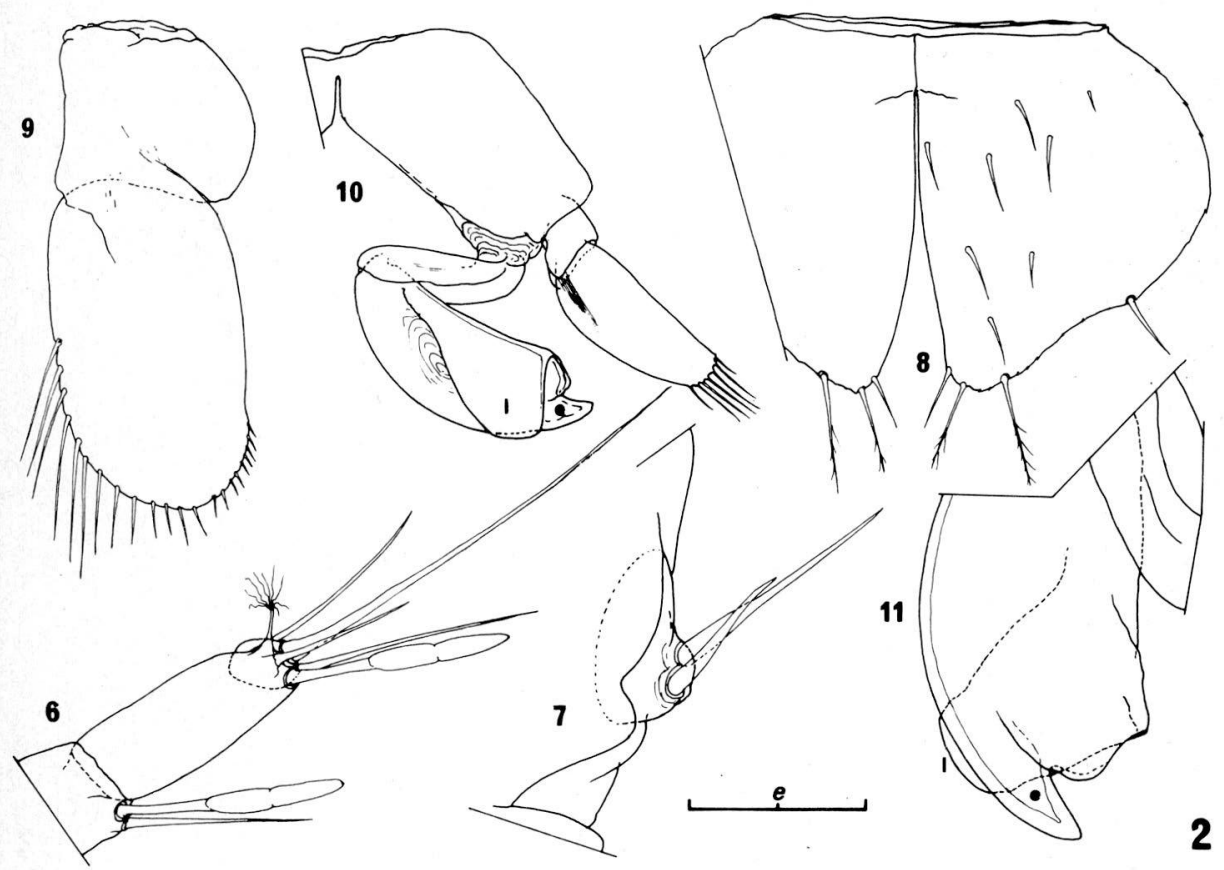

Fig. 6. Extrémité d'une antennule de la 9 de $13,9 \mathrm{~mm}$; $=100 \mu$

Fig. 7. Troisième article de la hampe d'une antenne de la même, avec la squama à 2 épines; $\mathrm{e}=$ $200 \mu$

Fig. 8. Pléopodes II de la même, face sternale; $\mathrm{e}=400 \mu$

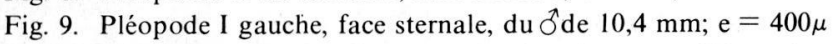

Fig. 10. Pléopode II gauche, face sternale, du même; $\mathrm{e}=400 \mu$

Fig. 11. Extrémité de l'endopodite du pléopode II droit du même, face tergale; e = éperon; $1=$ lamelle accessoire; $\mathrm{e}=200 \mu$

1. M. africana (Monod, 1945), espèce-type (Bull. I.F.A.N., 7, 101; sub Stenasellus africanus): taille: $11 \mathrm{~mm}(\hat{\partial}), 12 \mathrm{~mm}(q)$; puits à Man (Côte-d'Ivoire), bassin du fleuve Sassandra.

2. M. guinensis (Braga, 1950), (An. Fac. Ciên. Porto, 35, 50; sub Stenasellus guinensis): espèce géante dont seule la $+(18,5 \mathrm{~mm})$ est connue; puits de l'Administration de Gabu Sara = Nova Lamego (Guinée-Bissau), bassin de la rivière Géba.

3. M. studiosorum Sket, 1969, (Bull. Sci. Univ. Ljubljana, A, 14, 386): très petite espèce ( $\delta$ de 3,7 et $q$ de $5 \mathrm{~mm}$ ), trouvée dans des sources à Kédougou (Sénégal), bassin de la Gambie.

4. M. laticarpa (Birstein, 1972), (Int. J. Speleol., 4, 9; sub Stenasellus laticarpus): espèce de taille moyenne ( $\delta$ de 10,1 et $q$ de $10,5 \mathrm{~mm}$ ), capturée dans un puits à Sikasso (Mali), haut bassin du Niger. 


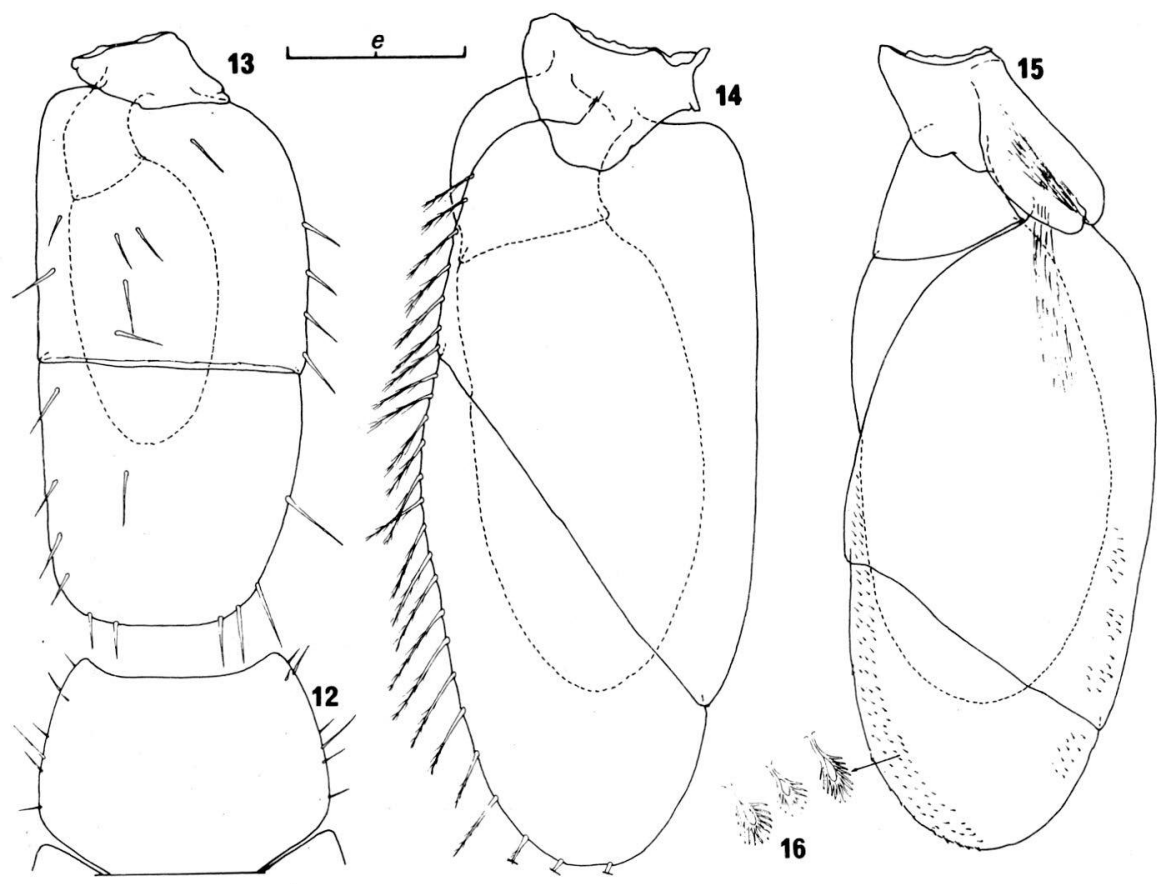

Fig. 12. Céphalon du de $10,4 \mathrm{~mm}$, vue tergale; $\mathrm{e}=1000 \mu$

Fig. 13. Pléopode III gauche, face sternale, du même; e $=666 \mu$

Fig. 14. Pléopode IV gauche, face sternale, du même; $\mathrm{e}=400 \mu$

Fig. 15. Pléopode V gauche, face sternale, du même; $\mathrm{e}=400 \mu$

Fig. 16. Ecailles cuticulaires auriculées de l'exopodite du précédent; $\mathrm{e}=66 \mu$

5. M. gardei n.sp.: haut bassin de l'Oued Saoura.

La diagnose du genre avait été donnée par Lanza (Monit. zool. ital., 74, 238-239, 1966), elle a été révisée (Magniez, 1974, p. 54) après la découverte des espèces $n^{\circ} 3$ et 4 . On peut maintenant la résumer ainsi:

- Sténasellides au corps allongé, un peu élargi au niveau des péréionites médians. Tergites des pléonites I et II très développés en longueur $(+\mathrm{de} 1 / 2 \mathrm{du}$ péréionite VII), un peu moins larges que ce dernier. Pléopodes I $\delta$ avec protopodite sans rétinacle. Endopodite du pléopode II ô biarticulé, avec article proximal long, formant le manubrium du distal grâce à une articulation genouillée mobile. Article distal en forme de lame ovalaire, pratiquement plane et pourvue sur sa marge externe d'une lamelle mobile plus petite, rabattue sur sa face sternale. Pléopodes II $q$ généralement indépendants ou avec une courte suture secondaire proximale. Exopodite des pléopodes IV avec suture interarticulaire très oblique et une rangée continue de soies sur la marge interne des deux articles. Exopodite des pléopodes $V$ lamellaire plus large et 


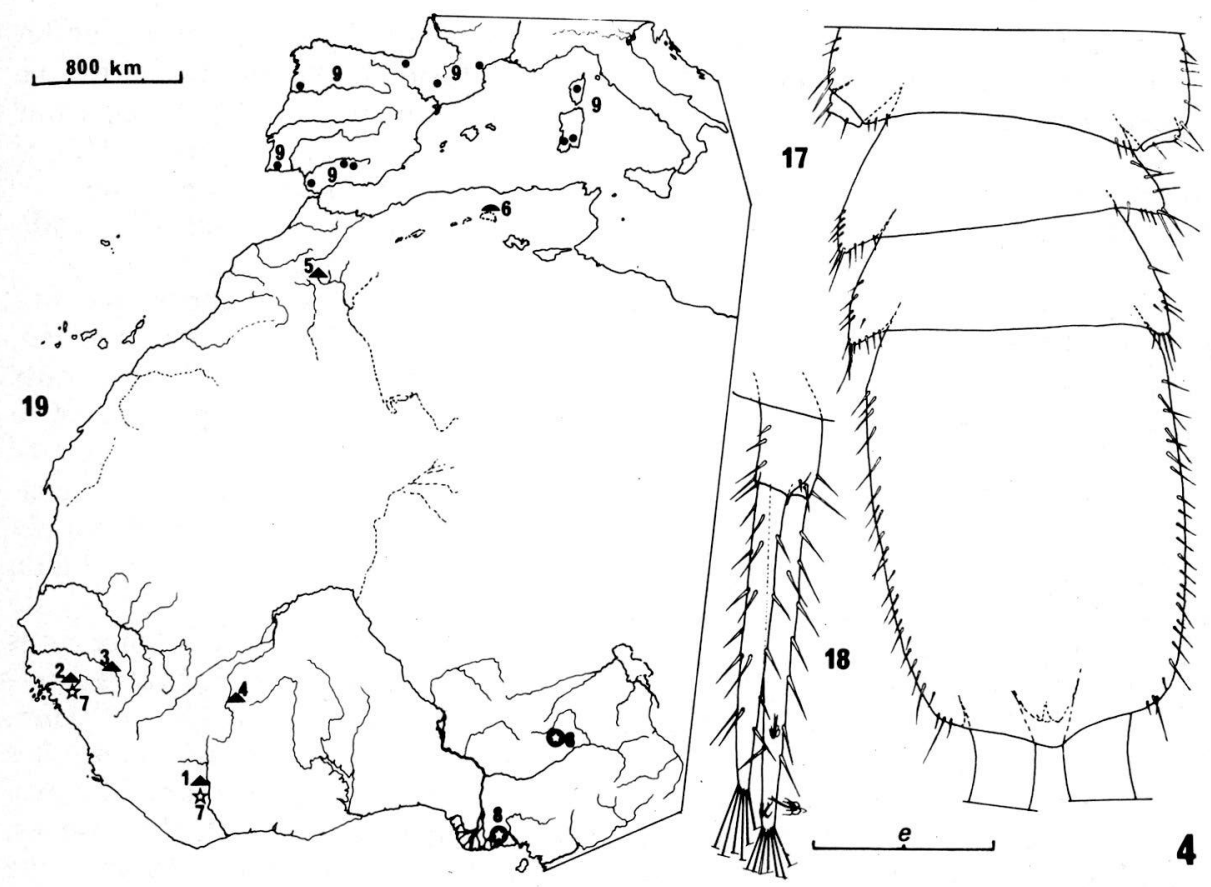

Fig. 17. Pléon du de $10,4 \mathrm{~mm}$, vue tergale; $\mathrm{e}=1000 \mu$

Fig. 18. Uropode gauche du précédent; $\mathrm{e}=1000 \mu$

Fig. 19. Carte schématique d'Afrique et d'Europe occidentales avec les stations des Sténasellides connus: $1=$ Magniezia africana $($ Monod, 1945), $2=$ M. guinensis $($ Braga, 11950), $3=M$. studiosorum Sket, 1969, $4=$ M. laticarpa (Birstein, 1972), $5=M$. gardei n.sp. du Maroc sud-oriental, $6=$ Johannella purpurea Monod, 1924, 7 = Parastenasellus chappuisi $(\mathrm{Remy}$, 1938), 8 = espèces du genre Metastenasellus Magniez, 1966, $9=$ espèces du genre Stenasellus Dollfus, 1897, car.emend. (11 espèces du Portugal, d'Espagne, de France méridionale, Corse, Sardaigne, Toscane).

plus long que son endopodite, avec une suture interarticulaire oblique. Uropodes presque aussi longs que le pléotelson.

Remarques biogéographiques: assez largement répandus dans les eaux souterraines d'Europe méridionale, avec le genre Stenasellus Dollfus (14 espèces disséminées du Portugal à la Bulgarie, plus 2 espèces asiatiques du Turkménistan et du Cambodge) et le genre Balkanostenasellus Cvetkov, 1975 ( 2 espèces de Bulgarie-Yougoslavie), les Sténasellides se retrouvent avec une diversification beaucoup plus importante en Afrique nord-tropicale et équatoriale: genres Magniezia Lanza, 1966 (4 espèces) et Parastenasellus Magniez, 1966 (1 espèce) du bouclier nigérien, genre Metastenasellus Magniez, 1966 ( 5 espèces dont une en cours de description, du bouclier congolais), genre Stenasellus s.str. d'Afrique orientale (4 espèces de Somalie-Kénya). Dans l'intervalle de ces deux aires 
de peuplement, un vaste hiatus géographique existait. C'est tout juste si un représentant de la famille (Johannella purpurea Monod, 1924) avait été mis en évidence dans une source thermale à M'Sila (180 km au S-E d'Alger), mais une seule $\delta$ fut capturée et l'espèce n'a pu être retrouvée depuis (Monod, 1972), si bien que l'on ignore si ses affinités sont plutôt africaines qu'européennes.

Bien que relativement distantes les unes des autres $(500$ à $800 \mathrm{~km})$, les localités des 4 espèces de Magniezia antérieurement connues (Man, Nova Lamego = Gabu Sara, Kédougou et Sikasso) sont néanmoins proches à l'échelle du continent. Elles se répartissent naturellement à la périphérie du massif guinéen, château d'eau de l'Afrique occidentale, d'où divergent de nombreux cours d'eau. La nouvelle espèce, $M$. gardei apparaît donc très éloignée (2200 à $2500 \mathrm{~km}$ ) de l'aire primitivement attribuée au genre (Magniez, 1974, p. 54). Elle en semble surtout isolée par une immense étendue désertique. Les Sténasellides étant depuis fort longtemps strictement inféodés aux eaux souterraines continentales, interstitielles ou karstiques, il est évident que la présence d'un peuplement sud-marocain de Magniezia ne peut s'expliquer dans le contexte climatique de la région: il s'agit d'un peuplement relicte. Son existence nous permet de penser que ces Sténasellides ont autrefois colonisé les eaux souterraines d'une vaste zone ouest-africaine, de l'Atlas marocain à la Côte d'Ivoire, de l'Atlantique à l'Adrar et au Hoggar, mais dans des conditions climatiques permettant l'existence d'un couvert végétal, d'un réseau hydrographique permanent et de nappes phréatiques pérennes, y compris au niveau des interfluves. L'hiatus actuel entre les faunes sud-européennes et africaines semble donc davantage lié au développement récent du climat aride qu'à une absence originelle de ces Crustacés dans le sous-sol des régions sahariennes intermédiaires. Il est intéressant de rappeler à cette occasion la découverte par le Prof. P. Remy, dans cette même région, d'un autre Isopode aquatique souterrain ( $\mathrm{Mi}$ crocerberus remyi Chappuis, 1953, de l'interstitiel de l'Oued Todgha, affluent du Ghéris): elle appelle les mêmes remarques que celles faites pour $M$. gardei. Manuscrit terminé le 4 janvier 1978.

\section{RESUME}

Cette nouvelle espèce a été découverte dans les eaux de la grotte Kef Aziza, sur le versant saharien du Haut-Atlas, au Sud-Est du Maroc. Magniezia gardei n.sp. appartient au même genre que quatre espèces précédemment décrites des eaux phréatiques de la région guinéenne. Aussi peut-on penser que cette espèce marocaine serait une relicte d'une ancienne faune aquatique hypogée, autrefois répandue dans toute l'Afrique occidentale et maintenant isolée par le développement du climat aride. 


\section{BIBLIOGRAPHIE}

BIRSTEIN, J. A., 1972: Une nouvelle espèce africaine du genre Stenasellus (Crustacea Isopoda A sellota) du bassin du Niger. Int. J. Speleol., 4, 9-18.

BRAGA, J. M., 1950: Sur deux Stenasellus (Crust. Isopoda) de la Guinée portugaise. An. Fac. Ciên. Porto, 35, 50-56.

CHAPPUIS, P. A., 1935: Un nouvel Isopode psammique du Maroc: Microcerberus remyi. Vie et Milieu, Fr., IV/4, 659-663.

CVETKOV, L., 1975: Sur la position systématique des Stenasellinae (Crustacea Asellota) de Bulgarie. Acta Zool. Bulgar., 1, 9-22.

LANZA, B., 1966: Stenasellus pardii sp.n. della Somalia e note sistematiche su gli Stenasellinae (Crustacea Isopoda). Monit. zool. ital., 74, 221-256.

MAGNIEZ, G., 1966: Contribution à la systématique des Stenasellinae d'Afrique (Crustacés Asellotes). Int. J. Speleol., 2, 173-190.

1974: Données faunistiques et écologiques sur les Stenasellidae (Crustacea Isopoda Asellota des eaux souterraines). Ibid., 6, 1-80.

1975: Observations sur la biologie de Stenasellus virei (Crustacea Isopoda Asellota des eaux souterraines). Ibid., 7, 79-228.

MONOD, Th., 1924: Sur quelques Asellides nouveaux des eaux douces de l'Afrique du Nord. Bull. Soc. Hist. nat. Afr. Nord, 15, 327-226, 7 pl.

- 1945: Un nouveau Stenasellus ouest-africain. Bull. Inst. fr. Afr., 7, 101-114.

- 1972: Sur les localités originales de deux Crustacés Isopodes algériens cavernicoles. Ann. Spéléol. Fr., 27, 177-181.

SKET, B., 1969: Eine neue Art der Stenasellinae (Isopoda, Asellota) aus Senegal. Bull. Sci. Univ. Ljubljana, A, 14, 386-387. 